\title{
FACTORIZATION OF OPERATORS ON BANACH SPACE
}

\author{
MARY R. EMBRY
}

\begin{abstract}
In this paper it is shown that if $D$ and $E$ are continuous linear operators on a Banach space $X$, then the following are equivalent: (i) $B$ is a right factor of $A$, (ii) $B$ majorizes $A$ and (iii) the range of $B^{*}$ contains the range of $A^{*}$.
\end{abstract}

In [1] Douglas proves the following:

Theorem (Douglas). If $A$ and $B$ are operators ${ }^{1}$ on a Hilbert space $X$, then the following are equivalent:

(i) $A=B C$ for some operator $C$ on $X$,

(ii) $\left\|A^{*} x\right\| \leqq k\left\|B^{*} x\right\|$ for some $k \geqq 0$ and all $x$ in $X$,

(iii) range $A \subset$ range $B$.

An obvious question to ask is whether this theorem generalizes to the case in which $X$ is an arbitrary Banach space. (In this case we interpret (ii) as $\left\|A^{*} x^{*}\right\| \leqq k\left\|B^{*} x^{*}\right\|$ for all $x^{*}$ in $X^{*}$, the adjoint space of $X$.) The answer to this question is negative, for in [2] Douglas gives an example (which we include later in this paper) of operators $A$ and $B$ for which (iii) is true but (i) is false.

A second, less obvious, question to ask is whether Douglas' theorem remains valid for adjoints of operators on Banach space. The purpose of this note is to present a proof of the following:

Theorem 1. Let $D$ and $E$ be operators on a Banach space $X$. The following conditions are equivalent:

(i') $D=F E$ for some continuous linear transformation $F$ : range $E \rightarrow X,{ }^{2}$

(ii') $\|D x\| \leqq k\|E x\|$ for some $k \geqq 0$ and all $x$ in $X$,

(iii') range $D^{*} \subset$ range $E^{*}$.

Let us note first that this theorem indeed generalizes Douglas' theorem. To see this, let $A=D^{*}$ and $B=E^{*}$, which is possible since every Hilbert

Received by the editors July 10, 1972 and, in revised form, August 31, 1972.

AMS (MOS) subject classifications (1970). Primary 47A99.

Key words and phrases. Factorization of operators, majorization of operators, range inclusion of operators.

${ }^{1}$ By an operator we mean a continuous linear transformation.

${ }^{2}$ In general $F$ is defined only on the closure of the range of $E$.

(c) American Mathematical Society 1973 
space operator is an adjoint. Then the second and third statements in the two theorems are identical. Condition (i') in Theorem 1 becomes (i") $A^{*}=F B^{*}$ for some continuous linear transformation $F$ : range $B^{*} \rightarrow X$. But since $X$ is a Hilbert space $F$ has a continuous linear extension $G$ on $X$ so that $A^{*}=G B^{*}$. Thus $A=B G^{*}$ which retrieves (i) of Douglas' theorem.

It is also worthy of note that Theorem 1 remains valid if $D: X \rightarrow Y$ and $E: X \rightarrow Z$, where each of $X, Y$, and $Z$ is a Banach space. In that case statement (i') becomes $D=F E$, where $F$ : range $E \rightarrow Y$. This generalization of Theorem 1 may be proven exactly as Theorem 1 will be proven.

To facilitate the proof of Theorem 1 we first prove a lemma, which is a generalization of a theorem of Mac Nerney [3, Theorem 1] and which was later rediscovered by Shmulyan [4, Lemma 3].

Lemma 1. Let $A: X \rightarrow Y$ be a continuous linear mapping of Banach spaces. Then $x^{*} \in \operatorname{range} A^{*}$ if and only if there exists a real number $k$ such that $\left|x^{*}(x)\right| \leqq k\|A x\|$ for all $x$ in $X$.

Proof. In one direction we argue as follows: if $x^{*}=A^{*} y^{*}$, then $\left|x^{*}(x)\right| \leqq\left\|y^{*}\right\|\|A x\|$. In the other direction we assume that $\left|x^{*}(x)\right| \leqq$ $k\|A x\|$ for all $x$ in $X$ and define $L$ :range $A \rightarrow$ \{complex numbers by $L(A x)=x^{*}(x) . L$ is well defined, continuous and linear and thus by the Hahn-Banach Theorem has continuous linear extension $y^{*}$ on $Y$. Therefore $y^{*}(A x)=L(A x)=x^{*}(x)$ for all $x$ in $X$, or equivalently, $A^{*} y^{*}=x^{*}$, showing that $x^{*} \in$ range $A^{*}$.

Proof of Theorem 1. To see that (i') and (ii') are equivalent we argue as follows: if $D=F E$, then $\|D x\| \leqq\|F\|\|E x\|$ for all $x$ in $X$ and if $\|D x\| \leqq k\|E x\|$ for all $x$ in $X$, we define $F$ by $F E x=D x$. The majorizing condition forces $F$ to be continuous and linear on the range of $E$.

We shall now show that (ii') and (iii') are equivalent. If $\|D x\| \leqq k\|E x\|$ for all $x$ in $X$, then for each $x^{*}$ in $X^{*}$ we have $\left|\left(D^{*} x^{*}\right)(x)\right| \leqq k\left\|x^{*}\right\|\|E x\|$ for all $x$ in $X$, which by Lemma 1 shows that $D^{*} x^{*} \in \operatorname{range} E^{*}$. Thus (ii') implies (iii').

Now assume that (iii') holds. For each positive integer $n$ define $M_{n}=$ $\left\{x^{*}: x^{*} \in X^{*}\right.$ and $\left|x^{*}(D x)\right| \leqq n\|E x\|$ for all $x$ in $\left.X\right\}$. Since (iii') holds, Lemma 1 implies that $\bigcup_{n} M_{n}=X^{*}$. It is easily checked that each $M_{n}$ is closed in $X^{*}$. Consequently the Baire Category Theorem applies and there exist an integer $n$, a positive number $r$ and an element $x_{0}^{*}$ of $M_{n}$ such that $x^{*} \in M_{n}$ whenever $\left\|x^{*}-x_{0}^{*}\right\| \leqq r$. Thus if $\left\|x^{*}\right\| \leqq r$, we have for each $x$ in $X$,

$$
2\left|x^{*}(D x)\right| \leqq\left|\left(x^{*}-x_{0}^{*}\right)(D x)\right|+\left|\left(x^{*}+x_{0}^{*}\right)(D x)\right| \leqq n\|E x\|+n\|E x\| .
$$

From this last inequality we see that $\left|x^{*}(D x)\right| \leqq n\|E x\|$ for all $x$ in $X$ 
whenever $\left\|x^{*}\right\| \leqq r$. Thus for all $x^{*}$ and all $x$ we have $\left|x^{*}(D x)\right| \leqq$ $(n / r)\|E x\|\left\|x^{*}\right\|$ and consequently $\|D x\| \leqq(n / r)\|E x\|$ for all $x$ in $X$, completing the proof.

The author is grateful to Professor Douglas for his permission to include the following counterexample:

Douglas' counterexample. Let $X$ be a Banach space, $N$ a subspace of $X$, and $Y$ the set of bounded functions on the integers so that $f(n)$ is in $X$ for $n \leqq 0$ and $f(n)$ is in $X / N$ for $n>0 . Y$ is a Banach space with respect to $\|f\|=\sup \|f(n)\|$. Consider the operators $A$ and $B$ on $Y$ defined by

$$
\begin{aligned}
& (A f)(n)=f(n) \text { for } n=1 \quad \text { and } \\
& =0 \text { for } n \neq 1 \\
& \begin{aligned}
(B f)(n) & =\pi f(0) & & \text { for } n=1 \\
& =f(n-1) & & \text { for } n \neq 1
\end{aligned}
\end{aligned}
$$

where $\pi$ is the natural map from $X$ to $X / N$. Then range $A \subset$ range $B$. Assume that there exists an operator $C$ on $Y$ such that $A=B C$. Let $D_{1}$ be the map from $X / N$ to $Y$ and $D_{2}$ the map from $Y$ to $X$ defined by

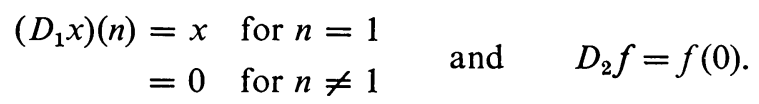

Then $E=D_{2} C D_{1}$ is a map from $X / N$ to $X$ such that $I-E \pi$ is a bounded projection of $X$ onto $N$. Thus if we choose $N$ to be a subspace for which no bounded projection exists, then we arrive at a contradiction and see that there exists no operator $C$ on $Y$ for which $A=B C$.

Let us now reconsider briefly the implications involved between conditions (i), (ii) and (iii) of Douglas' theorem when $X$ is an arbitrary Banach space. It should be obvious that (i) implies each of (ii) and (iii). By a category argument similar to that used in the proof of Theorem 1, we could show that condition (iii) implies condition (ii). Thus Douglas' counterexample also gives an example in which (ii) is true but (i) is false. The only remaining question concerning the relation of these three conditions is whether (ii) implies (iii). Theorem 1 gives an affirmative answer to this problem if $X$ is reflexive; however, it is an open question whether (ii) implies (iii) in the general case.

ADDENDUM. After the completion of this paper the author received a communication from Richard Bouldin, University of Georgia, indicating that he has found an example of operators on Banach space for which condition (ii) of Douglas' theorem holds, but condition (iii) does not hold.

\section{REFERENCES}

1. R. G. Douglas, On majorization, factorization and range inclusion of operators on Hilbert space, Proc. Amer. Math. Soc. 17 (1966), 413-415. MR 34 \#3315. 
2. R. G. Douglas, Addendum for "Majorization, factorization, and range inclusion" (unpublished).

3. J. S. Mac Nerney, Investigation concerning positive definite continued fractions, Duke Math. J. 26 (1959), 663-677. MR 22 \#8107.

4. Ju. L. Smul'jan, Two-sided division in the ring of operators, Mat. Zametki 1 (1967), 605-610=Math. Notes 1 (1967), 400-403. MR 36 \#729.

Department of Mathematics, University of North Carolina, Charlotte, North Carolina 28213 